\title{
Shoah, letteratura e zona grigia in Partigia
}

Le polemiche suscitate da Partigia, il libro dello storico Sergio Luzzatto che indaga sulle vicende della prima Resistenza nella Valle d'Aosta dell'autunno-inverno del 1943, in cui fu coinvolto più o meno direttamente Primo Levi, erano più che prevedibili, forse anche previste e volute dall'autore. II libro tocca vari punti nevralgici, sia per gli storici della Resistenza che per i critici e i biografi di Primo Levi, entrambi campi di studio ancora capaci, dopo lunghi decenni, di appassionare, addirittura di "ossessionare”, per usare una parola cara a Luzzatto e per cui è stato pesantemente criticato. In particolare, l'ossessione si manifesta attraverso il tentativo di svelare cause e effetti dell'uccisione di due giovani antifascisti, Fulvio Oppezzo e Luciano Zabaldano, da parte della piccola banda di neo-partigiani di cui anche Levi fece parte, pochi giorni prima della sua cattura. La convergenza tra le due ossessioni dichiarate in Partigia - per la Resistenza e per Levi - la commistione in esse tra storia e letteratura, e tra una gamma di casi umani e di situazioni storiche con una fortissima carica di ambiguità e di simbolismo, hanno dato risultati esplosivi, per certi versi preoccupanti, ma per altri sintomatici del momento in cui ci troviamo nella storia e nella memoria collettiva italiana. Proprio a causa di queste reazioni e per via delle tensioni già presenti nel libro che le hanno fatte scattare, il caso risulta tanto più affascinante per chi studia l'Italia da fuori, per chi da tempo cerca di capire quali valenze possano ancora avere questi residui di storia del Novecento nel calderone culturale e mediatico dell'inizio del nuovo millennio. Quali linee di forza convergono in questo uso e riuso di 
un passato così particolareggiato, o meglio "microstorico", e nel dibattito più o meno edificante che ne è seguito?

Cercando di prescindere dai toni polemici, vorrei soffermarmi brevemente su tre degli aspetti portanti del libro e del caso Partigia, nel tentativo di pesare il significato dell'operazione e di collocarlo all'interno di alcune genealogie culturali e intellettuali.

\section{Levi, la Shoah e la Resistenza}

Innanzitutto Partigia va inserito nella lunga e travagliata storia dei rapporti culturali e storiografici che si instaurarono nell'Italia del dopoguerra tra la Resistenza e ciò che viene comunemente chiamata oggi (ma soltanto da pochi anni in qua) la Shoah. Per tutta una prima fase della memorializzazione del genocidio, quando ci fu un silenzio quasi totale nella società e nella cultura italiana in generale, o meglio di una diffusa presenza di frammenti di memoria e di testimonianza che però non riuscirono ad attirare l'attenzione dei guardiani della cultura, la deportazione degli ebrei nei campi di sterminio venne letta e immaginata attraverso le lenti della deportazione politica di anti-fascisti o partigiani nei campi di concentramento di Mauthausen e simili. Valga come illustrazione del fenomeno il percorso del raggruppamento più attivo $\mathrm{e}$ influente nella memorializzazione e nella rappresentanza dei reduci dei campi nazisti in Italia, l'ANED: denominato già nell'immediato dopoguerra "Associazione nazionale degli ex-deportati politici nei campi nazisti”, soltanto nel 1968 la sigla venne ufficialmente rielaborata in "Associazione nazionale ex deportati politici e razziali nei campi di concentramento nazisti”. Altrettanto eloquente è l'esempio del film di Carlo Lizzani del 1961, L'oro di Roma, che chiude con una drammatica rievocazione del rastrellamento di più di mille ebrei nel ghetto di Roma del 6 ottobre 1943. Da una parte, l'eroina del film, Giulia, interpretata da Anna Maria Ferrero, sceglie di non abbandonare la propria famiglia e la comunità ebraica nel momento della distruzione, e finisce quindi nelle mani delle SS rimanendo vittima del genocidio; dall'altra l'eroe Davide (Gérard Blain) sceglie un'altra strada, ma in qualche modo anche una strada equivalente e parallela, difendendo allo stesso tempo la propria identità ebraica e quella italiana dandosi alla macchia, alla Resistenza. Quindi Shoah e Resistenza, due storie in una mitica visione d'armonia, con forte impegno di sacrifico e di combattimento antifascista da entrambe le parti. Dalla stessa 
matrice derivò il progetto del Memoriale dei morti italiani nei campi nel BlocCo 21 ad Auschwitz I, inaugurato nel 1980, e tanto criticato a partire dal 2008 da Giovanni De Luna e altri per la sua vena di "vulgata resistenziale", stile anni settanta, sovrapposta alla memorializzazione della Shoah.

Strano effetto della fine della guerra fredda e della prima Repubblica tra fine anni '8o e anni '9o, dunque, è stato il capovolgimento quasi totale di questo rapporto: non più la Shoah letta attraverso la lente della Resistenza, ma la lotta antifascista vista come un elemento tra tanti di un rifiuto del crimine più terribile del secolo, della Shoah. Oppure la Resistenza trascurata, oscurata da una attenzione ubiqua al genocidio. Alessandro Portelli, nel suo libro di storia e memoria orale sulle Fosse Ardeatine, L'ordine è stato già eseguito [1999], nota subito una certa confusione tra alcuni giovani di oggi, che credono che la storia di via Rasella e della rappresaglia riguardi più che altro la storia degli ebrei e l'Olocausto.

Con Partigia non è del tutto chiaro dove Luzzatto intenda indirizzare l'ago della bilancia tra Shoah e Resistenza, ma i due strati di storia si sovrappongono e s'intersecano in continuazione, esplicitamente e implicitamente. In parte, Luzzatto mantiene una specie di mezza finzione, cioè che in fondo Primo Levi abbia poco a che fare con la sostanza delle sue ricerche, con il perno storico e morale del libro. Effettivamente, se il libro e le ricerche di Luzzatto partono da Levi, dalla sua lunga ossessione per Levi, a un certo punto Partigia lascia le orme del futuro scrittore-testimone nel momento in cui abbandona la Valle d'Aosta per seguire il destino terribile che lo porterà ad Auschwitz: I'attenzione rimane strettamente puntata verso l'ambito dei partigiani tra Amay e Casale Monferrato, tra i protagonisti che uscirono vivi o morti da quelle montagne prima, durante e dopo quell'autunno del '43: "Questa è una storia di partigia in valle d'Aosta, non di deportati in campo di sterminio» (126). E ancora, insistentemente, il libro si caratterizza come «una storia della Resistenza per raccontare la storia della Resistenza» (17) e descrive Levi come "comprimario», come "reagente etico» (19) alle problematiche aggrovigliate della primissima Resistenza.

Ma partire da Levi - e da Vanda Maesto e Luciana Nissim, e dalla rete di rifugiati e neo-resistenti ebrei del 1943 - vuole dire comunque inquadrare e leggere la Resistenza attraverso il filtro della Shoah, delle deportazioni e dei crimini nazisti, e della gamma degli anti-semitismi fascisti, saloini e altro. Per di più, all'interno di una generale ascesi della Shoah a categoria ermeneutica per spiegare tutte le storie del Novecento, passare attraverso la biografia e l'opera di Levi, diventato ormai quasi un mito della voce del sopravvissuto e 
dell'autorità etica, sondare i suoi singoli testi per scoprire messaggi nascosti, segreti intorno alla Resistenza, non può non condizionare la ricerca storica che ne deriva, creando un ibrido affascinante, caratteristico di un momento forse nuovo nel rapporto Resistenza-Shoah e nella memoria collettiva del '9oo in Italia. Questo senz'altro aiuta a spiegare la forza e anche l'ambiguità del libro.

\section{Letteratura e storia}

Colpisce il fatto che Partigia è un libro di ricerca storica intrisa di letteratura. Ripetutamente, Luzzatto ricorre a evocazioni letterarie, a metodi della critica letteraria e della lettura di testi letterari "a chiave", oltre che a slanci narrativi che vanno dal pathos e dall'intimità delle tragedie personali dei vari "personaggi principali" presentati in un "dramatis personae" all'inizio del libro, a vari drammatici colpi di scena, spesso e volentieri messi insieme a racconti del tempo presente, che vedono e seguono lo storico nelle vicende attuali delle sue ricerche, tra sopralluoghi sul territorio, speculazioni su Google, e incontri romanzeschi con protagonisti invecchiati o parenti insospettati. II tutto crea una vera forza nella scrittura e nel coinvolgimento del lettore che fuoriesce dallo stile e dalla retorica accademica, con chiare affinità con la migliore storiografia "popolare" del mondo anglosassone. II rischio principale di una tale scrittura, si sa, è l'intrusione della categoria dell'individuo, delle storie e dei casi dei singoli a oscurare altri livelli di comprensioni storiche - e umane. Non è tanto il caso del pathos delle vittime ebree in Partigia a dare un esempio di questa forza ma anche dei rischi relativi, quanto il caso straordinario - da soggetto cinematografico, da letteratura, come dice apertamente Luzzatto - di Edilio Cagni, il traditore di Levi e della sua banda, che il libro segue nel dopoguerra in una vita rocambolesca tra fascismo redivivo, oro nascosto, tribunali, galere e servizi segreti americani. II libro lascia alla nostra fantasia immaginare il possibile destino di Cagni dopo gli anni 5o, ma con questa suspense romanzesca - Luzzatto lo chiama «il suo romanzo criminale» (282) siamo lontani anni luce dalla studio pacato e delicato sia della Resistenza che della Shoah da cui nasce il libro.

Oltre allo storico che sogna di essere anche narratore - vecchia vocazione in verità, colorita in epoca recente da influenti tendenze all'auto-racconto caratteristiche di varie scuole di antropologia, biografia, e storia - in Partigia 
troviamo anche vari spunti addirittura di citazione e di critica letteraria, come una sorta di innesto con la ricerca storica solida e tradizionale. Ci sono digressioni e riflessioni in alcuni punti chiave del libro sulla letteratura resistenziale di Pavese, di Calvino, di Fenoglio, di Rigoni Sterm e di Rosetta Loy. E verrebbe volentieri da aggiungere riferimenti a Pasolini e al fratello Guido, vittima di scontri tra partigiani, oppure al Bertolucci della Strategia del ragno in cui un traditore dell'antifascismo (come furono ritenuti dai compagni Oppezzo e Zabaldano) viene (come loro) innalzato nel dopoguerra a eroe della patria. $\mathrm{Ci}$ sono poi commenti sul ruolo del testimone "letterario" rispetto al testimone giuridico - soprattutto quando troviamo Levi ad assumere entrambi i ruoli negli stessi mesi del dopoguerra - ma anche in generale contrasti tra i ruoli e gli assunti ermeneutici di scrittori, giudici e storici. L'effetto è quello di creare una sensazione di ansia intorno al ruolo dello storico, in particolare dello storico della Resistenza - evidente anche nei discussi accenni a Pansa - che viene risolta attraverso delle "verità" scoperte nella letteratura e nel racconto. Viene da pensare a un altro libro, parallelo per certi versi a Partigia, in cui una crisi della memoria della Resistenza e delle sue certezze viene messa in luce in un ibrido di storia orale, di ricerca storiografica archivistica e di racconto, II disperso di Marburg di Nuto Revelli [1994].

Al centro di questa complessa e affascinante operazione storico-letteraria, sta l'ossessione di Luzzatto per l'opera leviana e il tentativo di rileggere alcuni dei suoi testi - un paragrafo del Sistemo periodico, le stesure e le varianti di Se questo è un uomo, il rapporto medico sull'igiene ad Auschwitz III scritto insieme a Leonardo De Benedetti, Se non ora, quando? e l'impianto "resistenziale", e soprattutto alcune poesie di Levi, inclusa quella che dà il titolo al libro di Luzzatto - tutti riletti attraverso l'ottica di quei giorni di Levi in Valle d'Aosta e della sua (dichiarata) angoscia per l'uccisione di due "compagni”, Oppezzo e Zabaldano. Senza entrare nel merito delle singole letture dei testi - alcuni hanno criticato Luzzatto per citazioni parziali o fuori contesto, ma il percorso segna indubbiamente una matrice presente e ricorrente nell'immaginario e nella memoria leviani - è però da notare la trappola di una lettura troppo "storicizzante", troppo a chiave e quindi unidimensionale, di testi come quelli di Levi, in cui non c'è tanto l'ambiguità di tanta letteratura moderna o modernista, quanto una pluralità di livelli di scrittura, un uso diffuso dell'ironia, leggera e analitica allo stesso tempo, e della trasfusione e della modulazione di esperienza in riflessione e comprensione etica, e quindi non soltanto storica. Se l'archivio non dà certezze sulle circostanze precise dell'uccisione di Oppezzo e Zabaldano - e le critiche più taglienti contro Luzzatto forse ven- 
gono da quelli che notano l'assenza al cuore del libro, cioè il fallito tentativo di risolvere per certo il "giallo" del perché sono stati giustiziati - poco ci fa pensare che la letteratura, le apparenti "verità" della letteratura, perfino della letterature testimoniale, possano colmare la lacuna.

Tutto questo sforzo narrativo-letterario in Partigia è un altro elemento che aiuta a spiegare l'eco che il libro ha suscitato. Visto in una prospettiva lunga, conferma la straordinaria forza dell'ibrido storico-letterario nell'interrogarsi sul passato, un elemento di un certo postmodernismo impegnato forse, ma anche segno di una certa vocazione per ibridazioni del genere nella letteratura italiana, che risale almeno fino al Manzoni della Storia della colonna infame e che si manifesta poi nelle varie inchieste storico-letterarie di Leonardo Sciascia, nelle "microstorie" di Carlo Ginzburg, ma anche in Portelli, in Revelli e altri. Non sempre in questa genealogia la storia e la letteratura mantengono un equilibrio stabile tra di loro.

\section{Zona grigia}

Luzzatto scava nell'opera di Primo Levi e con grandi colpi di scena "scopre" quelle che gli sembrano alcune verità profonde sullo stato d'animo di Levi e sulla pesante eredità delle sue esperienze resistenziali. Interessante notare, però, che quasi non gli viene di nominare l'ultimo libro di Levi sulla Shoah, cioè I sommersi e i salvati del 1986, tanto meno il saggio che forse ha avuto più risonanza in esso, La zona grigia. C'è comunque motivo di pensare che nel concetto morale e storiografico della "zona grigia" troviamo il cuore del progetto di Partigia, sia nella sua accezione leviana dei Sommersi e i salvati che nell'accezione, per certi versi abusiva rispetto al pensiero di Levi, diffusa in tutto il campo della storiografia della Resistenza da De Felice a Pavone e da lì in giù. In effetti, anche se non viene esplicitamente nominato né citato, la zona grigia (o meglio La zona grigia) sta senz'altro dietro la dichiarazione culminante di metodo e di principio - fortemente "pavesiana" oltre che leviana - posta alla fine della prefazione di Partigia:

Sarebbe rassicurante pensare che in una guerra (tanto più in una guerra civile) il nemico sia sempre e comunque fuori di noi. $\mathrm{E}$ che, una volta vinto il nemico, risolto sia il problema del male. Sarebbe molto rassicurante, ma sarebbe troppo comodo. Visto da vicino, la guerra civile italiana - dove nessuno dovrebbe faticare, almeno retrospettivamente, a scegliere il proprio campo: I'uno essendo il campo dell'umanità e del diritto, l'altro quello della disumanità e 
dell'abuso - racconta una storia diversa. Insieme con la storia del bene, l'impagabile bene della lotta contro il nazifascismo, racconta la storia di un male insondabile, il male da cui nessun essere umano, nemmeno il migliore, può dirsi totalmente affrancato. Così, tra il bianco e il nero, numerose si rivelano qui le tonalità del grigio. A volte, la storia dei partigia ha il fascino semplice dei contrasti. Più spesso, ha la verità complicata delle sfumature. (21; corsivo mio)

Il brano va citato per esteso per capire qualcosa di fondamentale importanza in Partigia: la guerra civile italiana è qui raccontata (o meglio la guerra "racconta" se stessa) attraverso il grigio, attraverso la zona grigia; non tanto o non soltanto quella dell'attendismo e della scelta di non scegliere, quanto il concetto originario di Levi dei Sommersi: il concetto della situazione storica che impone all'individuo scelte necessarie e impossibili, moralmente compromesse a priori, a cui, per dirla con Levi, almeno nei casi estremi e terribili dei Sonderkommandos oppure di Chaim Rumkowksi, "re del ghetto" di Lodz, "non conosco tribunale umano a cui delegarne la misura» [Levi 1997, II/1023]. In parte, Luzzatto tenta di mettere Levi stesso - sicuramente il referente della frase sopra "nessun essere umano, nemmeno il migliore» - in questa zona del grigio, colpevole di un atto eccessivo con la sua banda, ma non necessariamente da essere giudicato in tribunale. Ma si sa che Levi comunque e sempre si autocollocava nella zona grigia, tra i piccoli privilegiati del sistemaAuschwitz (fosse soltanto per quei mesi invernali passati all'interno del laboratorio del Buna invece che nel freddo polare della Alta Slesia), implorando ai suoi lettori più volte di non considerarlo né un santo né un eroe, per il puro caso (o peggio) di essere sopravvissuto al vortice. Se Partigia non riesce del tutto a mettere a fuoco il grigio di Levi, lo stesso vale in fondo per i due ragazzi, per Oppezzo e Zabaldano, che riescono molto inafferrabili, semmai grigi semplicemente nella loro sprovvedutezza. E Cagni, come abbiamo visto, sta nettamente dalla parte del nero, tra il nero fascista e film noir.

Rimane come punto focale dell'indagine del mondo grigio di questo racconto della guerra civile italiana un altro fascista, un'altra figura in cui Luzzatto scopre un altissimo grado di ambiguità e di contraddittorietà, e di nuovo una sovrapposizione tra guerra, Fascismo, Resistenza e Shoah. È la figura di «Cesare Augusto Carnazzi, avvocato, prefetto di Aosta e poi di Asti» (6), capo e responsabile di Cagni e dell'infiltrazione da parte dei fascisti della banda di Levi, "funzionario antisemita se non ... antisemita militante» (188), che con grande stupore Luzzatto scopre di essere anche un salvatore di ebrei, nella fattispecie nel 1944 Ladilsao Gerber di una famiglia polacca rifugiata con altri ebrei a Gandino, quasi alla stregua di alcuni "Giusti fra le nazioni", tra cui sei abitanti di Gandino riconosciuti addirittura a Yad Vashem (192). Carnazzi 
sembra incarnare il grigio, il nero contaminato dal bianco nella stessa persona, il singolo atto che lo salvi dalla nostra condanna più totale.

Ma anche qui ci sono trappole da evitare, soprattutto la trappola di un grigio relativistico, il grigio che c'è in ognuno di noi, e che quindi discolpa in qualche modo tutti. Non è detto che Luzzatto cada nella trappola, ma alcuni dei suoi lettori certo potrebbero. Cosa di cui nella prefazione citata sopra sembra riconoscere il rischio con l'affermazione ansiosa che tutti noi dobbiamo sapere da che parte sta il giusto. Lo stesso concetto leviano di "zona grigia" già nel 1986 è stato subito ripreso e distorto, iniziando il suo lungo percorso verso una categoria storica anti-ideologica invece che etico-politica. Alberto Cavaglion ha ricordato lo stupore di Levi di fronte all'uso, o meglio all'abuso, da parte di Giorgio Bocca della categoria del grigio al momento dell'uscita dei Sommersi, per spiegare, e per "perdonare" in qualche modo, le oscure vicende di guerra dell'austriaco ed ex-soldato nazista Kurt Waldheim [Cavaglion 2004]. Da ricordare nel caso di Waldheim, ma anche di Carnazzi e altri presentati all'insegna del grigio "universale", per così dire, l'orrore di Levi nella Zona grigia di fronte alla favola, che chiama addirittura "rivoltante», della cipollina dei Fratelli Karamazov: "quale mostro umano non ha mai donato in vita sua una cipollina...? Quel singolo atto di pietà subito cancellata non basta certo ad assolvere...» [Levi 1997, II/1035]. Per Levi, il grigio ci serve a vedere meglio e a capire nell'insieme il bianco e il nero, il bene e il male; non a oscurarli, tanto meno a confonderli.

\section{Bibliografia}

Portelli A. 1999, L'ordine è stato già eseguito, Roma: Donzelli.

Revelli N. 1994, Il disperso di Marburg, Torino: Einaudi 1994.

Levi P. 1997, Opere, voll. 2, Torino: Einaudi.

Cavaglion A. 2004, Primo Levi tra i sommersi e i salvati, "Lo straniero", 7 (48): 40-49. 